\title{
Juggling slow and fast science
}

To the editor - The slow-science movement started as a call for a healthier and better science culture, described by Lisa Alleva ${ }^{1}$ in 2006 as a 'rewarding and pleasurable' way of doing science ${ }^{1}$. The movement has been gaining momentum over the past years, and, in an opinion piece published in November 2019, Uta Frith ${ }^{2}$ argued that fast science is "bad for both science and scientists." Only a few months later, the World Health Organization declared the COVID-19 outbreak a public health emergency of international concern. The impacts of SARS-CoV-2 accelerated science in an unforeseeable manner. A year later, more than 97,000 scientific manuscripts about COVID-19 could be found on the PubMed database and $50+$ vaccines were in clinical development (https://covid-nma.com/ vaccines/mapping/), suggesting that 2020 brought the scientific community to an even faster pace. What does the speed of science amidst the pandemic mean for young scientists?

The current academic system promotes quantity over quality, rewards fast-paced science, and ostracizes scientists who do not fit this profile 3 . In this 'publish or perish' culture, young scholars, especially those competing for permanent positions, are expected to publish frequently in high-impact journals ${ }^{4}$.

The speed of science is directly related to power structures and inequalities ${ }^{5}$. Fast science reinforces gender and racial disparities, disfavouring underrepresented groups $^{6}$. It affects the mental health of young researchers, especially those burdened with impostor syndrome ${ }^{7}$. For some scholars, a more diverse, more collaborative, and healthier academic environment is only possible through slow science ${ }^{5,6}$.

As early-career childless Latina biologists, the pandemic brought us a sense of urgency to contribute to scientific knowledge about COVID-19 and the motivation to work harder and faster. However, we now find ourselves in a dilemma: while any decrease in productivity is questioned by senior collaborators, who remain oblivious to the need to adjust their expectations of our work rate, a boost in productivity gets a side-eye from young peers who assume that fast science is inherently flawed and that slow science is the only way towards high-quality research ${ }^{8}$. Besides, engaging in fast science is sometimes perceived as an insult to female peers who had their lives overturned by the pandemic and can no longer engage in scholarly work. Sadly, we learned that slow scientists can be quick to judge, and in a somewhat strange plot twist, we now feel guilty for the 'privilege' of being overworked.

While advocating for a more diverse, inclusive, and healthier science, we feel that the dichotomy created between slow and fast science has added another layer of anxiety to an already disquieting scenario. We agree that some science cannot and should not be rushed ${ }^{9}$, but COVID-19 has also taught us that some science cannot and should not be slowed. During the pandemic, the assumption that one speed fits all, whether fast or slow, is revealed to be more hurtful than helpful.

As we juggle expectations and scrutiny, we remind the scientific community that the slow science movement can only exist as a collective enterprise. We underline that attempts to design a normative speed of science is inherently against the principles of the slow science movement. We urge the scientific community to respect and value the peculiarities of different fields and the working rhythms, styles, and circumstances of different scientists. Only then will scientists have the chance and the support needed to flourish and contribute at their own pace.

Luciana Leite ${ }^{1,2,3}$ and

Luisa Maria Diele-Viegas (DD,4凶

${ }^{1}$ School of Psychological Science, Oregon State University, Corvallis, Oregon, USA. ${ }^{2}$ Biology Institute, Federal University of Bahia, Salvador, Bahia, Brazil. ${ }^{3}$ Kunhã Asé Network of Women in Science, Salvador, Bahia, Brazil. ${ }^{4}$ Biology Department, University of Maryland, College Park, Maryland, USA.

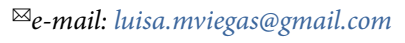

Published online: 11 March 2021

https://doi.org/10.1038/s41562-021-01080-1

References

1. Alleva, L. Nature 443, 271 (2006).

2. Frith, U. Trends Cogn. Sci. 24, 1-2 (2020).

3. Fischer, J., Ritchie, E. G. \& Hanspach, J. Trends Ecol. Evol. 27, 473-474 (2012).

4. Salo, P. \& Heikkinen, H.L.T. Confero https://doi.org/10.3384/ confero.2001-4562.181130 (2018).

5. Martell, L. Forum Qual. Soc. Res. https://doi.org/10.17169/fqs15.3.2223 (2017).

6. Mountz, A. et al. ACME 14, 1235-1259 (2015).

7. Jaremka, L. M. et al. Perspect. Psychol. Sci. 15, 519-543 (2020).

8. Roche, D. G. et al. Sci. Total Environ. 696, 134000 (2019)

9. Owens, B. Nature 495, 300-303 (2013).

Acknowledgements

We thank the Kunhã Asé Network of Woman in Science for raising the discussion that originated the idea for this piece. During the development of this piece, L.L. was funded by Coordenação de Aperfeiçoamento de Pessoal de Nível Superior (CAPES).

Competing interests

The authors declare no competing interests. 